\title{
La generación de conocimiento en Trabajo Social: percepción de graduadas y graduados de dos programas de Magíster en Trabajo Social de Chile
}

\author{
Yenny Edith Figueroa Reyes ${ }^{1}$; Isis Chamblás García²; Gabriela Rubilar Donoso ${ }^{3}$
}

Recibido: 23/11/2016 / Revisado: 18/01/2017 / Aceptado: 06/03/2017

Resumen. El presente artículo da cuenta de uno de los ámbitos abordado en la investigación que busca describir las prácticas investigativas y el tipo de conocimiento generado a través de las tesis de postgrado, así como la percepción que tienen los graduados del aporte de su investigación de postgrado en la generación de conocimiento para la disciplina y para las políticas sociales. Se consideró el caso del Magister de Trabajo Social y Políticas Sociales (MTSPS) de la Universidad de Concepción y del Magister en Trabajo Social (MTS) de la Pontificia Universidad Católica de Chile. La investigación se centra tanto en los trabajos de grado como en los graduados de los últimos 10 años (2005 a 2014) en ambos programas, así como en los documentos de tesis. La muestra final resultó de 32 (MTSPS) y 28 (MTS) graduados, fue una muestra de tipo accidental, dado que se envió el instrumento al grupo total; para la selección de tesis, se eligió una muestra probabilística simple de 40 casos en cada programa; para el análisis de las prácticas investigativas, derivada de las tesis de grado, se elaboró una matriz de análisis y para la medición de las variables asociadas a las miradas de los graduados acerca de su trabajo de investigación, se utilizó la encuesta en su modalidad on-line.

Los principales resultados indican una percepción positiva del impacto de sus trabajos de investigación en la política y el Trabajo Social, sin embargo en la práctica indican insuficiente publicación y/o seguimiento de la utilización del conocimiento generado en estos trabajos de postgrado.

Palabras clave: Aportaciones investigación; Postgrado; Trabajo Social; investigación y desarrollo profesional; generación de conocimiento.

\section{[en] Knowledge creation in social work: the views of graduates of two social work master's programmes in Chile}

\begin{abstract}
This article reports on one of the areas addressed in the research aimed at describing research practices and the kind of knowledge created through postgraduate theses, as well as graduates' perceptions of the contribution made by their postgraduate research in creating knowledge for the discipline and for social policy. The cases of the Master's Degree in Social Work and Social Policy (MTSPS) from Universidad de Concepción and of the Master's Degree in Social Work (MTS) from the Pontificia Universidad Católica in Chile were taken into account.

The research focuses both on master's theses and on the graduates of the last ten years (2005 to 2014) in both programmes, as well as on dissertation papers. The final sample was made up of 32 (MTSPS) and 28 (MTS) graduates. It was a convenience sample, since the instrument was sent to the whole group. For the selection of dissertations, a simple probabilistic sample of 40 cases was chosen for each programme. An analysis matrix was produced for the analysis of research practices, deriving from the degree master's theses, and the online form of the survey was used to measure the variables associated with graduates' views of their research work.

The main results indicate a positive perception of the impact of research into policy and social work. However, in practice there is insufficient publication and/or monitoring of the use of knowledge created through these postgraduate works.
\end{abstract}

Key words: research contributions; postgraduate; social work; professional research and development; knowledge creation.

Universidad de Concepción, Chile.

yenny.figueroa.r@gmail.com

Universidada de Concepción. Chile.

ichambla@udec.cl

Universidad Católica de Chile

grubilad@gmail.com 
Sumario: Introducción. 1. Metodología. 2. Resultados. 2.1. Aporte de los trabajos de investigación de postgrado a la política social. 2.2. Divulgación y/o utilización del conocimiento generado a partir de los trabajos finales para la graduación de los estudiantes. 2.3. Percepción del efecto de la formación de postgrado y la participación en "espacios" de la política pública. 2.4. Efecto de la formación de postgrado en la trayectoria profesional de los graduados de Magíster 3. Discusión. 4. Conclusiones. 5. Referencias bibliográficas.

Cómo citar: Figueroa Reyes, Y. E.; Chamblás García, I.; Rubilar Donoso, G. (2018) La generación de conocimiento en Trabajo Social: percepción de graduadas y graduadas de dos programas de Magíster en Trabajo Social de Chile, en Cuad. trab. soc. 31(2), 407-416.

\section{Introducción}

Reconociendo que los cambios sociales y culturales experimentados en las últimas décadas han llevado a que la disciplina del Trabajo Social indague y busque cada vez de manera más óptima e innovadora la forma de responder a esos nuevos desafíos, el desarrollo de la investigación aparece como un eje fundamental para consolidar y fortalecer este conocimiento (Campos, 2006).

La investigación en Trabajo Social ha estado presente desde los orígenes de la profesión y su aporte como la creación de conocimiento han sido capaces de nutrir y aumentar los fundamentos teóricos de la profesión en muchas de sus áreas, considerándola como un articulador base para la existencia de nuevos y mejores métodos para conocer la realidad (Aylwin, Forttes y Matus, 2004; Shaw, 2015, p. 102).

Bajo este contexto, en este artículo se concibe la relación entre investigación e intervención social como parte de un mismo continuo que ha estado presente - aunque con otras denominaciones - desde los orígenes de la profesión. Algunos autores, como Kisnerman (1985), Heller (2002) y Matus, Aylwin y Forttes (2004) y Grassi (1995), plantean que desde el inicio del Trabajo Social los profesionales han hecho investigación, pero esta suele encontrarse "encapsulada o silenciada" y, por lo mismo, se desarrolla de forma marginal o desde ámbitos que cuentan con escasa difusión e incidencia pública. Desde esta perspectiva, la investigación no sería antagónica a la intervención sino más bien una dimensión que se ha de potenciar y complementar, reconociendo con ello el carácter complejo de ambos procesos y sus múltiples interrelaciones $(\mathrm{Ru}-$ bilar, 2009).

Tanto la propia profesión como los actuales y diversos cambios y complejidades sociales, que se han presentado a través del tiempo han dado lugar a la búsqueda de opciones múltiples e innovadoras, ya sea en investigación social como en estrategias innovadoras de intervención social. En este contexto, surge parte de la investigación realizada en el ámbito de las políticas públicas y sociales y el desarrollo, y en particular la participación del Trabajo Social con relación al diseño, implementación y evaluación de políticas sociales (Valdés y Espina, 2011).

Todo el análisis previamente realizado, en cuanto a: la creación de los programas de postgrado en Trabajo Social, la importancia de la investigación como generador de conocimiento para la disciplina, el enlace entre investigación y política social que posibilitaría una serie de nuevas estrategias y metodologías en el área, el perfeccionamiento del egresado y el efecto en su desarrollo profesional y laboral, hacen relevante profundizar en el aporte de los trabajos de investigación realizados desde estos programas a la disciplina, las políticas sociales y también en especial a los propios graduados.

En el Trabajo Social, tanto la intervención como la investigación nutren indiscutiblemente la disciplina, permitiéndoles a sus profesionales una mayor preparación y un conocimiento respecto de su quehacer cotidiano. La creación de conocimiento a través de la investigación ha sido capaz de nutrir y aumentar los fundamentos teóricos de la profesión en muchas de sus áreas, considerándola como un eje fundamental para la existencia de nuevos y mejores métodos para conocer la realidad, siendo este un foco central de estudio del Trabajo Social (sujeto y objeto de observación). Así autores, como Hessen (1956), respecto del origen del conocimiento humano, menciona que: "a partir de él se logran enfrentar la conciencia y el objeto, generando un dualismo de sujeto y objeto como parte de la esencia del conocimiento" (p.50). 
Tanto para el Trabajo Social como para otras disciplinas, la producción de conocimiento es una exigencia para su legitimidad y desarrollo como tal (Grassi, 2007). La investigación científica propicia en gran medida que la curiosidad del conocimiento genere resultados válidos y fiables que se pueden refrendar y comparar; así mismo, la investigación puede ser calificada como el camino y la meta de búsqueda y producción de conocimiento que debe realizarse de forma organizada y sistemática (p.29). Cívicos, y Hernández, (2007), citando a Jara (1998), refieren que el Trabajo Social como profesión: "cuenta con un caudal enorme de experiencias y de saber implícito e intuitivo que se pierde, lamentablemente, si no se comunica, explicita o se transforma en conocimiento científico" (p. 46). En este sentido, menciona que:

La falta de sistematización y transmisión de esos saberes debilita la disciplina y la hace dependiente de otras áreas de conocimiento"; es preciso, por lo tanto, "recuperar la voz para configurar una identidad más potente, sistematizar lo que se hace es un primer paso para no dejar que esas experiencias se pierdan y dejen de generar nuevos conocimientos y avances sociales (p.52).

[Lo anterior] hace especialmente relevante la necesidad de que constantemente se promueva un ejercicio de reflexión y metaconocimiento en torno a la forma como se ha desarrollado la investigación y los aportes que esta ha dado a las distintas disciplinas, la necesidad de contar con información idónea y actualizada para la toma de decisiones en pro del mejoramiento profesional y académico (Calderón, Chavarría y Garro, 2010, p.18).

Desde este punto, es posible preguntarse entonces: ¿qué tipo de conocimiento se está produciendo en los programas de postgrado? ¿Quiénes son los graduados de los programas de postgrado que realizan estas investigaciones? ¿Qué tipo de investigaciones se están desarrollando? ¿Qué se está realizando con este conocimiento?

En este contexto nace esta propuesta de investigación que tiene como propósito un acercamiento al conocimiento generado a través de las tesis de postgrado, y en forma particular, el aporte de las mismas en las políticas y programas sociales visto desde los propios graduados y sus percepciones al respecto.

\section{Metodología}

La investigación es de tipo descriptiva- con un enfoque cuantitativo; se buscaba conocer la mirada de los propios graduados respecto del aporte a nivel de generación de conocimiento de sus tesis y las características de estas prácticas investigativas. Para lo anterior se definió una muestra del 50 por ciento de los documentos de tesis y del 100 por ciento de los graduados en los últimos 10 años (2005 a 2014) en cada uno de los programas considerados, lo que corresponde a 59 en el caso del Magister de Trabajo Social de la Pontificia Universidad Católica (MTS) y 74 en el caso del Magister en Trabajo Social y Políticas Sociales de la Universidad de Concepción (MTSPS). Finalmente, respondió un total de 32 graduados de la segunda y 28 de la primera.

Se utilizó la técnica de encuesta, en la modalidad on line. Se eligió esta modalidad -consciente de los problemas de retorno- porque era la mejor forma de poder acceder a los graduados, quienes se encontraban en distintas ciudades del país. La encuesta se diseñó considerando la medición de las variables centrales de esta investigación; Percepción del aporte de su investigación a la generación de conocimiento, a la política social y a su desarrollo profesional; así como la difusión del conocimiento generado en sus tesis de grado y el rol que les compete a ellos en el desarrollo de nuevas investigaciones. Dicho instrumento fue sometido a pruebas de validez de contenido a través de análisis de expertos.

En otro ámbito, se abordaron las características de las prácticas investigativas a través del análisis documental de las tesis de grado, aspecto no abordado en este artículo.

\section{Resultados}

En los programas de Magister analizados estudian mayoritariamente mujeres y las respuestas de los encuestados mantienen esta tendencia, el 80 por ciento (de 32) en el Magister de Trabajo Social y Políticas Sociales de la Universidad de Concepción y el 64 por ciento (de 28) en el Magister de Trabajo Social de la Pontificia Universidad Católica de Chile; se trata mayoritariamente de una población joven, que inició su formación de postgrado, en promedio, a los 28 años (27 años en el Magister en Trabajo Social y 29 años en el Magis- 
ter de Trabajo Social y Políticas Sociales). La profesión de origen de los graduados de ambos programas es mayoritariamente la de asistente o trabajador/a social, y en menor proporción la de sociólogo/a, periodista o historiador/a; la mayoría reconoce una trayectoria de perfeccionamiento en diplomaturas y postitulaciones; así mismo algunos casos indican que, una vez egresados de sus maestrías, han iniciado estudios de doctorado (12 por ciento en el Magister de Trabajo Social y Políticas Sociales de la Universidad de Concepción y 40 por ciento en el Magister de Trabajo Social de la Pontificia Universidad Católica de Chile).

\subsection{Aporte de los trabajos de investigación de postgrado a la política social}

La percepción de los graduados respecto del aporte, que sus trabajos de tesis pudieron generar a la disciplina o a la política social, es en su mayoría que el trabajo realizado sí representó un aporte al Trabajo Social (cerca del 60 por ciento en el Magister de Trabajo Social y Políticas Sociales y el 71,4 por ciento del Magister en Trabajo Social), y en menor proporción aunque también reconocen que representa un aporte a la política social (el 40,6 por ciento en Magister de Trabajo Social y Políticas Sociales versus el 43 por ciento en Magister en Trabajo Social) y a otras áreas (el 40,6 por ciento).

Los aspectos destacados para fundamentar dicha contribución se refieren a los aportes a la "disciplina del Trabajo Social" y a la "crítica del programa y/o tema en estudio" para el caso de los graduados del Magister en Trabajo Social, mientras que los graduados del Magister de Trabajo Social y Políticas Sociales reconocen un aporte en la "descripción de los programas y/o temas" asociados a estos programas sociales. Finalmente, en ambos programas se reconoce que el aporte a la política pública se presenta en alguna de sus etapas (análisis, generación, implementación y/o evaluación).

Por otra parte, al solicitar una evaluación de su tesis de grado como aporte innovador al diseño, ejecución y/o análisis de la política social, solo el 43,7 por ciento (26 casos del total de graduados encuestados)lo atribuye a su trabajo y el 39 por ciento declara no saberlo, situación que arroja interrogantes respecto del análisis crítico y proyección de los resultados que realizan los graduados al momento de concluir su trabajo de investigación.

\subsection{Divulgación y/o utilización del conoci- miento generado a partir de los trabajos fi- nales para la graduación de los estudiantes}

Los espacios de "difusión" del conocimiento han sido variados. Sin embargo solo cerca del 40 por ciento de los graduados de ambas maestrías declaran haber publicado los resultados de su investigación en una revista científica. La mayor parte de ellos sí reconoce su aporte en otros espacios, como: clases de pregrado y postgrado, este hecho se presenta más elevado en el Magister en Trabajo Social que en el Magister de Trabajo Social y Políticas Sociales (el 53,6 y el 18 por ciento respectivamente); también en "reuniones técnicas" para público profesional son otra instancia de difusión frecuentemente utilizada (el 25 por ciento en el Magister en Trabajo Social y el 31,3 por ciento en el Magister de Trabajo Social y Políticas Sociales). También existe, aunque en menor medida, la participación en "congresos" y en "conferencias" (el 18 y el 12,5 por ciento respectivamente). Finalmente, se señala que un promedio del 29,6 por ciento declara no haber utilizado los resultados de sus investigaciones en ninguna de las anteriores opciones de difusión.

En términos globales, solo cerca del 20 por ciento de los graduados creen que "los conocimientos generados han sido difundidos y aprovechados satisfactoriamente", versus el 42 por ciento que opinan que los resultados obtenidos de sus investigaciones no se han aprovechado ni difundido oportunamente; este hecho incluso genera interrogantes respecto a las razones que subyacen a estas respuestas, así como la reflexión para cada programa acerca de las acciones implementadas para lograr mayor motivación en los estudiantes, así como para generar espacios e instancias para difundir y aprovechar los resultados de estos trabajos de las tesis. Un alto porcentaje de los encuestados de ambos programas considera que es responsabilidad compartida, tanto del propio graduado (el 86,7 por ciento) como del magister (el 78,3 por ciento). El 28,3 por ciento cree que son las instituciones receptoras de los resultados (organizaciones vinculadas a la investigación realizada) quienes tienen esta responsabilidad.

Por otra parte, la mayoría de estos graduados (el 80 por ciento en el Magister de Trabajo Social y Políticas Sociales y el 39 por ciento en el Magister en Trabajo Social) reconocen que sus tesinas no fueron complementadas con 
nuevas investigaciones, aunque el 54 por ciento de este último sí reconoce un trabajo posterior, vinculado a dicha materia.

Las principales dificultades mencionadas para publicar, corresponden a "la falta de tiempo" (el 45,8 por ciento en el Magister de Trabajo Social y Políticas Sociales frente al 37,5 por ciento en el Magister en Trabajo Social); el "rechazo de sus publicaciones" (el 31,3 por ciento en este último), "el cambio de rubro profesional" y "la falta de interés en publicar" (el 20,8 por ciento en el Magister de Trabajo
Social y Políticas Sociales); y también se menciona "el desconocimiento de la forma de presentar y/o publicar un artículo científico", "la escasa vinculación con revistas científicas" y la "falta de apoyo para publicar" (el 12, 5 por ciento en el Magister en Trabajo Social). En estas tres últimas situaciones son los graduados de este último Magíster quienes presentan mayores porcentajes de respuestas, lo que se asocia al hecho que este magister no contempla exigencia de publicación para la graduación de sus estudiantes (véase Tabla 1).

Tabla 1: "Motivos por lo que no se realizan otras publicaciones"

\begin{tabular}{|l|l|l|l|}
\hline \multicolumn{1}{|c|}{ Motivos por los que NO se realizan otras publicaciones: } & \multicolumn{1}{|c|}{$\begin{array}{c}\text { MTSPS } \\
\text { (en \%) }\end{array}$} & $\begin{array}{c}\text { MTS } \\
\text { (en \%) }\end{array}$ & $\begin{array}{c}\text { TOTAL } \\
\text { (en \%) }\end{array}$ \\
\hline 1.Cambio de rubro profesional & 20,8 & 0,0 & 10,4 \\
\hline 2.Desconocimiento & 4,2 & 12,5 & 8,4 \\
\hline 3.Falta de interés en publicar & 20,8 & 12,5 & 16,7 \\
\hline 4.Falta de tiempo & 45,8 & 37,5 & 41,7 \\
\hline 5.Rechazado/a en publicación postulada & 8,3 & 31,3 & 19,8 \\
\hline 6.Trabajando en actual publicación & 0,0 & 6,3 & 3,2 \\
\hline Total $(\mathrm{n})$ & $(24)$ & $(16)$ & $(40)$ \\
\hline
\end{tabular}

Fuente: Elaboración propia

Lo anterior contrasta, en cierta medida, con el reconocimiento de los graduados de ambos programas, quienes consideran que luego del magister están más preparados para realizar investigación (el 90 por ciento y superior), se reconocen con "formación que les permite participar de equipos de investigación", y con la preparación para "realizar investigación de calidad y en su disciplina”. Estos resultados son muy relevantes (véase Tabla 2), considerando el énfasis investigativo que imparten los programas, y así también reconocen que "es parte de su rol profesional" difundir los resultados de las investigaciones (cerca del 80 por ciento responde "muy de acuerdo" y "de acuerdo) 
Tabla 2:"Percepción del rol investigador de los graduados por programa"

\begin{tabular}{|l|c|c|c|c|}
\hline & \multicolumn{2}{|c|}{ MTSPS } & \multicolumn{2}{c|}{ MTS } \\
\hline $\begin{array}{l}\text { Luego de sus estudios en el Ma- } \\
\text { gister: Usted considera que: }\end{array}$ & $\begin{array}{c}\text { Muy de acuerdo } \\
\text { (en \%) }\end{array}$ & $\begin{array}{c}\text { De acuerdo } \\
\text { (en \%) }\end{array}$ & $\begin{array}{c}\text { Muy de acuerdo } \\
\text { (en \%) }\end{array}$ & $\begin{array}{c}\text { De acuerdo } \\
\text { (en \%) }\end{array}$ \\
\hline $\begin{array}{l}\text { 1. La formación de postgrado reci- } \\
\text { bida le permite realizar investiga- } \\
\text { ciones en su disciplina }\end{array}$ & 43,8 & 36,8 & 42,3 & 53,6 \\
\hline $\begin{array}{l}\text { 2. Considera que tiene formación } \\
\text { para participar en equipos de inves- } \\
\text { tigación }\end{array}$ & 53,1 & 36,8 & 50,0 & 46,4 \\
\hline $\begin{array}{l}\text { 3. Cree que esta formación le } \\
\text { permite realizar investigación de } \\
\text { calidad }\end{array}$ & 37,5 & 53,1 & 42,3 & 50,0 \\
\hline $\begin{array}{l}\text { 4. La formación de postgrado le } \\
\text { permite desempenarse en un rol } \\
\text { profesional como investigador }\end{array}$ & 31,3 & 56,3 & 46,4 & 42,9 \\
\hline $\begin{array}{l}\text { 5. La formación de postgrado le } \\
\text { permitió incorporarse al mundo } \\
\text { académico }\end{array}$ & 25,0 & 21,9 & 46,4 & 25,0 \\
\hline $\begin{array}{l}\text { 6. Considera que ahora tiene alguna } \\
\text { responsabilidad de investigar en su } \\
\text { disciplina }\end{array}$ & 34,4 & 50,0 & 57,1 & 32,1 \\
\hline $\begin{array}{l}\text { 7. Considera más importante la } \\
\text { investigación en su disciplina }\end{array}$ & 53,1 & 31,3 & 42,3 & 17,9 \\
\hline $\begin{array}{l}\text { 8. Actualmente, Cree usted que es } \\
\text { parte de su rol profesional difundir } \\
\text { resultados de las investigaciones } \\
\text { realizadas }\end{array}$ & 53,1 & 31,3 & 60,7 & 28,6 \\
\hline Total & $\mathbf{( 3 2 )}$ & $\mathbf{( 3 2 )}$ & $\mathbf{( 2 8 )}$ & $\mathbf{( 2 8 )}$ \\
\hline
\end{tabular}

El total de cada fila no corresponde al 100 por cien, ya que en cada ,pregunta las categorías de respuesta eran 5 en total

Fuente: Elaboración propia

\subsection{Percepción del efecto de la formación de postgrado y la participación en "espacios" de la política pública}

Los graduados de ambos programas reconocen que, luego del egreso de sus respectivos magíster, han logrado incorporarse en algunas de las fases de la política social, por lo que se puede asumir que los estudios de postgrado posibilitan cambios y mejoras en la partici- pación de estos graduados en diversos ámbitos-fases de la política pública en general, tanto en la formulación de política pública (del 8,3 al 30 por ciento), como en la evaluación de alguna política social (del 13,3 al 51,7 por ciento). En general para todas las instancias-etapas de la política, los porcentajes de participación de los graduados aumentaron a casi más del 50 por ciento en ambos magister (véase Tabla 3). 
Tabla 3: "Participación en políticas públicas y/o programas sociales, Antes y Después del Magister"

\begin{tabular}{|c|c|c|c|c|c|c|}
\hline \multirow{2}{*}{$\begin{array}{l}\text { Participación en políticas públicas } \\
\text { y/o programas sociales: }\end{array}$} & \multicolumn{3}{|c|}{$\begin{array}{l}\text { ANTES DEL MAGISTER } \\
(\text { en } \%)\end{array}$} & \multicolumn{3}{|c|}{$\begin{array}{l}\text { DESPUES DEL MAGISTER } \\
(\text { en \%) }\end{array}$} \\
\hline & MTSPS & MTS & TOTAL & MTSPS & MTS & TOTAL \\
\hline $\begin{array}{l}\text { 1. Ha participado en la formulación, } \\
\text { implementación, análisis-evaluación } \\
\text { de Políticas Públicas y Sociales }\end{array}$ & 21,9 & 21,4 & 21,7 & 50,0 & 46,4 & 48,3 \\
\hline $\begin{array}{l}\text { 2. Ha participado en la formulación } \\
\text { de alguna Política Pública o Progra- } \\
\text { ma Social }\end{array}$ & 6,3 & 10,7 & 8,3 & 31,3 & 28,6 & 30,0 \\
\hline $\begin{array}{l}\text { 3. Ha participado en la implementa- } \\
\text { ción de alguna/s política/s públicas o } \\
\text { programas sociales }\end{array}$ & 31,2 & 28,6 & 30,0 & 53,1 & 46,4 & 50,0 \\
\hline $\begin{array}{l}\text { 4. Ha participado en la evaluación o } \\
\text { seguimiento de alguna política públi- } \\
\text { ca o programa social }\end{array}$ & 9,4 & 17,9 & 13,3 & 53,1 & 50,0 & 51,7 \\
\hline $\begin{array}{l}\text { 5. Considera que tiene un rol que } \\
\text { cumplir en la formulación, imple- } \\
\text { mentación, análisis-evaluación de } \\
\text { Políticas Públicas y Sociales }\end{array}$ & 6,3 & 7,1 & 6,7 & 46,9 & 57,1 & 51,7 \\
\hline Total (n) ${ }^{1}$ & (32) & (28) & $(60)$ & (32) & (28) & $(60)$ \\
\hline
\end{tabular}

Fuente: Elaboración propia

En síntesis al revisar lo anterior, podemos mencionar que la "participación en la formulación y evaluación de políticas públicas" aumentó considerablemente en los graduados de ambos programas, por lo que podemos afirmar que luego de graduarse, existiría una mayor visibilidad y acción en el área. Por otra parte, si bien la mayor cantidad de los encuestados cree tener el rol de agente implementador de la política social (el 63,3 por ciento), no es menor el porcentaje de casos que declara participar en el diseño o evaluación de la política o programa (el 33,3 por ciento), así como también quienes participan en la propuesta del tema a abordar (el 25 por ciento) y como profesional de apoyo externo a la política (el 26,7 por ciento).

\subsection{Efecto de la formación de postgrado en la trayectoria profesional de los graduados de Magíster}

A partir de lo informado por los propios encuestados, es posible mencionar que, después de egresar del programa de Magister, se denota el impacto en la inserción laboral, considerando el porcentaje de graduados que se encontra- ban trabajando: el 100 por cien en el caso del Magister de Trabajo Social y Políticas Sociales y el 96,4 por ciento Magister en Trabajo Social, versus el 60 por ciento y el 61 por ciento respectivamente, al inicio del programa, lo que puede dar cuenta del efecto del programa en la empleabilidad.

Por otra parte, también se observó un cambio en el tipo de instituciones donde se desempeñaban profesionalmente. Al momento de ingresar en el Magister de Trabajo Social y Políticas Sociales y en el Magister en Trabajo Social, la mayoría de sus estudiantes se encontraba trabajando en municipalidades (el 34,4 y el 14,3 por ciento respectivamente) y en fundaciones (el 25 y el 21,4 por ciento respectivamente). Durante el desarrollo del programa esta tendencia se mantuvo, no así a su término, cuando la mayoría porcentual en ambos casos inició el desempeño laboral en Universidades (lo que corresponde con el perfil académico que los programas buscan potenciar). Luego de egresar del Magister de Trabajo Social y Políticas Sociales, quienes trabajaban en universidades aumentaron del 9,4 por ciento al 28,1 por ciento; de igual modo se presentó 
para el Magister en Trabajo Social, que aumentó del 7,1 al 39,3 por ciento en la misma categoría. También hay un aumento en el porcentaje de graduados del Magister de Trabajo Social y Políticas Sociales que trabajaban en organismos gubernamentales (del 12,5 al 18,8 por ciento), y una disminución en quienes trabajaban en fundaciones, municipalidades $\mathrm{y}$ servicios de salud.

En algunos casos, los graduados mencionan haber tenido más de un trabajo a la vez, luego del egreso del Magister: en su gran mayoría asume funciones en docencia universitaria a la vez que se dedican paralelamente a otras funciones profesionales en instituciones como municipalidades, fundaciones y otras.

Por otra parte y luego del egreso, los graduados mejoraron las condiciones laborales: aumentó el porcentaje de casos de graduados con contrato indefinido y en jornada completa llegando al 90,6 por ciento en el Magister de Trabajo Social y Políticas Sociales y al 78,6 por ciento en los graduados del Magister en Trabajo Social (antes era el 71,9 y el 67,9 por ciento respectivamente). Cabe señalar que los trabajos a honorarios y a contrata son los menos recurrentes en este grupo de graduados de ambos programas. Por otra parte, un porcentaje considerable de los graduados informa que tuvieron un cambio favorable en sus remuneraciones, cerca del 78 por ciento en cada Magister versus el 12,5 por ciento del Magister de Trabajo Social y Políticas Sociales y el 17,9 por ciento del Magister en Trabajo Social que declaran no haber experimentado cambios.

Este panorama diverso y los cambios, también se observa en los cargos y funciones profesionales para los graduados del Magister de Trabajo Social y Políticas Sociales, las funciones que experimentaron más cambios fueron las de "coordinador profesional", que aumentó de 21,9 al 28,1 por ciento, y la de docencia, del 3,1 al 15,6 por ciento en cada caso; las que disminuyeron fueron las de asesor profesional, gestión, intervención profesional e implementador de política. En el caso del Magister en Trabajo Social, la función de asesor profesional, coordinación, gestión e implementador disminuyeron al egreso del programa. Las que se vieron aumentadas fueron las de dirección, del 3,6 al 10,7 por ciento), la de docencia del 7,1 al 17,9 por ciento, y la de investigador, del 10,7 al 21,4 por ciento.

En ambos programas se puede identificar un aumento del número de egresados, en car- gos y funciones asociadas a la docencia e investigación, lo que muestra que la formación de postgrado les entrega formación y herramientas para desempeñar estos nuevos roles profesionales.

\section{Discusión}

El presente trabajo, permite reflexionar, tal y como lo realiza Campos (2006), en la importancia de la formación de postgrado para los profesionales, ya que favorece la "construcción" de profesionales con alto nivel científico y técnico. Según lo mostrado en esta investigación, es relevante poder fortalecer el ingreso desde el pregrado a los estudios de postgrado, mejorando así la formación profesional y el quehacer de la disciplina Por el impacto que dicha formación tiene en la sociedad y también en la propia trayectoria profesional,

En términos de aportes a la disciplina del Trabajo Social, Palma (2010) asegura que muchos profesionales han logrado realizar una contribución a las políticas públicas, a través de la generación de conocimiento técnico o mediante la docencia, situación que es posible ver reflejada en el análisis de los trabajos de tesis de los programas de Magíster estudiados. Así también Calderón, Chavarría y Garro (2010) afirman que la investigación aporta al análisis, interpretación y trasformación de los problemas sociales que constituyen la demanda social y que, a su vez, justifican la definición, reorientación y fortalecimiento de políticas sociales, en este sentido los aportes metodológicos y de intervención, que reconocen los graduados a través de sus investigaciones y de los ámbitos de la política que señalan haber abordado, con miras a mejorar e innovar los contenidos y ejecución de estos programas sociales. Por otra parte, la participación de los graduados en espacios de políticas y programas sociales aumenta de manera importante luego del egreso del magíster, llegando casi al doble en ambos programas.

Una de las consideraciones más relevantes es que en ambos casos los graduados aumentan de forma considerable la percepción del rol que debe cumplir en las políticas sociales y sus procesos, especialmente la implementación y evaluación de las mismas. Palma (2010), recalca entre otros asuntos, que luego de los estudios de postgrados los graduados han podido incidir directamente en la elaboración, gestión, 
o en la evaluación de políticas y programas en los más diversos ámbitos de intervención, lo que conlleva a analizar el impacto que tiene este factor en la disciplina y la política social como tal. El aumento de percepción del rol investigativo, luego del egreso del magíster, y la alta consideración de los graduados respecto de su propio rol profesional para difundir los resultados de las investigaciones realizadas, se complementa con lo descrito por Palma, quien menciona que los programas de magíster tienen el propósito de formar profesionales especializados en investigación y con desarrollo de política social, donde se permita la producción de conocimiento mediante la investigación, y busca formar profesionales capaces de investigar y participar en todas las etapas de las políticas y programas sociales

En términos globales, la formación de postgrado también afecta la trayectoria profesional de los graduados. Los cambios laborales más importantes luego del egreso del programa son la mejora de las remuneraciones, la obtención de cargos directivos y lograr o fortalecer el ejercicio de la docencia universitaria, esto es consistente con lo expuesto también por Palma, quien afirma que el retorno a los profesionales luego de estudios de postgrado, es masivo y se caracteriza por un aumento en el nivel de responsabilidad de las funciones asumidas, lo que resultaría en una valoración de la for- mación recibida por parte de los organismos empleadores lo que incide en la empleabilidad.

\section{Conclusiones}

En la necesidad de fomentar el desarrollo de estrategias formativas y reconocer la importancia del ejercicio investigativo de forma sostenida, se plantea la interrogante respecto a la relevancia que adquiere el propio programa de Magíster en términos de la producción, promoción y divulgación de los resultados obtenidos desde las tesis de postgrado con el propósito de masificar y utilizar de mayor y mejor manera los aportes generados.

Otro de los desafíos más relevantes radica en que, según lo analizado, se plantean insuficientes investigaciones en términos de la política social propiamente, sugiriendo que más investigaciones apunten a este propósito, reconociendo al Trabajo Social como protagonista en todo el proceso de la política y no solo ejecutor, sino desde la formulación a la evaluación de dicha política.

Por otra parte, cabe preguntarse por la vinculación de los programas con los graduados, ya sea a través de un seguimiento, instancias de encuentro, dado que también se observó una baja en el número de respuestas de los profesionales respecto de la cohorte más antigua.

\section{Referencias bibliográficas}

Aylwin, N., Forttes, A. y Matus, T. (2004). La reinvención de la memoria. Indagación sobre el proceso de profesionalización del Trabajo Social Chileno 1925-1965. Revista de Trabajo Social, 388-401. Santiago de Chile: Pontificia Universidad Católica de Chile.

Calderón, A., Chavarría A. y Garro, J. (2010). La producción de conocimiento en la escuela de trabajo social a partir de los trabajos finales de graduación periodo 1998-2007. (Tesis. Universidad de Costa Rica, Escuela de Trabajo Social).

Campos, I. (2006). La formación profesional del posgrado en Trabajo Social en Costa Rica. Antecedentes, avances, direccionalidad y desafíos actuales. Revista Costarricence de Trabajo Social, 18, 1-13.

Cívicos, A. y Hernández, M. (2007). Algunas reflexiones y aportaciones en torno a los enfoques teóricos y prácticos de la Investigación en Trabajo Social. Revista Acciones e investigaciones sociales, 23, 25-55.

Grassi, E. (1995). La implicancia de la investigación social en la práctica del Trabajo Social. Revista Margen de Trabajo Social y Ciencias Sociales, 9, 43-54.

Grassi, E. (2007). Problemas de realismo y teoricismo en la investigación social y en el Trabajo Social. Revista Katálysis, 10, 26-36 Universidad Federal de Santa Catarina Santa Catarina, Brasil.

Heler, M. (2002). Filosofia social y Trabajo Social. Buenos Aires: Editorial Buenos Aires.

Hessen, J. (1956). Teoría del conocimiento. ( $8^{\circ}$ ed.). Buenos Aires: Austral/Espasa-Calpe.

Kisnerman N. et al. (1985). Introducción al Trabajo Social: Colección Teoría y Práctica del Trabajo Social. Edición ilustrada. Buenos Aires: Ediciones Humanitas. 
Palma, J. (2010). Magíster en Gestión y Política Pública: 15 años formando líderes para América Latina. Universidad de Chile. Recuperado de: http://www.mgpp.cl/2010/10/18/mgpp-15-anos-formando-lideres-para-america-latina/

Rubilar, G. (2009). ¿Cómo hacen investigaciones los trabajadores sociales? Una primera aproximación a las experiencias de investigación de una generación de profesionales chilenos. Revista de Trabajo Social Pontificia Universidad Católica de Chile, 76, 17-34.

Shaw, M. (2015). Nuevas miradas sobre la práctica del Trabajo Social desde un abordaje sistémico. Revista debate público, reflexión de Trabajo Social, 9.

Valdés, J. y Espina, M. (eds.). (2011). América Latina y el Caribe: la política social en el nuevo contexto. Enfoques y experiencias Uruguay: Ed. Alfredo Prieto-UNESCO. 